\title{
Steady state statistics of
} driven diffusions

\author{
Christian Maes, ${ }^{1}$ Karel Netočný, ${ }^{2}$ and Bram Wynants ${ }^{1}$ \\ ${ }^{1}$ Instituut voor Theoretische Fysica, K.U.Leuven, Belgiun * \\ ${ }^{2}$ Institute of Physics AS CR, Prague, Czech Republid
}

\begin{abstract}
We consider overdamped diffusion processes driven out of thermal equilibrium and we analyze their dynamical steady fluctuations. We discuss the thermodynamic interpretation of the joint fluctuations of occupation times and currents; they incorporate respectively the time-symmetric and the time-antisymmetric sector of the fluctuations. We highlight the canonical structure of the joint fluctuations. The novel concept of traffic complements the entropy production for the study of the occupation statistics. We explain how the occupation and current fluctuations get mutually coupled out of equilibrium. Their decoupling close-to-equilibrium explains the validity of entropy production principles.
\end{abstract}

\section{INTRODUCTION}

Thermodynamics already ceases to be exact under the microscope. That line of thought appeared a century ago, explicitly so in the introduction to Einstein's paper on the atomic hypothesis, [1]. The ensuing theory of Brownian motion started a dynamical fluctuation theory, and essentially the same mathematical model was further used by Onsager and Machlup, [2], to describe small macroscopic fluctuations around the relaxation to equilibrium. It has developed into the (sometimes called Lagrangian) approach to nonequilibrium statistical mechanics which is based on giving a weight to available trajectories in terms of an action that integrates a space-time local Lagrangian. Since then various methods have been developed to extract from that action functional physically relevant information about the steady state statistics.

In recent years there has been a revival of nonequilibrium fluctuation theory, with many contributions on various aspects of the problem. One class are static fluctuations referring to the statistics of macroscopic fluctuations upon a nonequilibrium stationary distribution; they have been mostly studied for driven lattice gases in the hydrodynamic regime, [3] , by generalizing the Hamilton-Jacobi method originally proposed in [4]. Related yet fundamentally different are the dynamical fluctuations concerning time-integrated observables. That is what this paper is about. We will introduce and also compare our present work with other recent approaches in Section IIC There has been a particular interest in the (integrated) current and entropy production fluctuations, also in the context of the celebrated fluctuation symmetry. It is nearly impossible to mention all the relevant references; as an example we include [5, 6, 7, 8, , 9, 10, 11].

An extension of the dynamical approach to the time-symmetric domain was initiated in [12, 13, 14]. In particular, it has been shown how the nonequilibrium fluctuations of the steady state occupation times are related to the mean entropy production, 14]. That relation proves useful for understanding the status and the limitations of entropy production principles in characterizing the steady state, [14, 15]. As further unfolded in [16] within the framework of Markov jump processes, a particularly simple and generic structure of dynamical fluctuations emerges when both the time-symmetric (occupation times) and the time-antisymmetric (current) fluctuations are observed jointly. Their correlation can be neglected in a close to equilibrium regime, where the familiar linear irreversible scheme based on the entropy production alone exists. However, the coupling between the time-symmetric and time-antisymmetric sectors becomes essential far from equilibrium. That can be seen as a fundamental reason for highly complex patterns of e.g. current fluctuations which arise when observing their marginal distribution alone. The main question remains to see some systematics in the fluctuation formulæ and to identify terms in the rate functions that have a general thermodynamic meaning. In order to complete that program the following examples will help as orientation.

The plan of the paper is as follows. In Section $\amalg$ we introduce a class of driven diffusion models on which we formulate our main questions and answers. In Section $\amalg$ we define the dynamical entropy, composed of a time-antisymmetric part (entropy flux) and a time-symmetric part (dynamical activity or traffic). Our main results come in Section IV where we show how entropy production functionals govern the dynamical fluctuations of diffusion processes. We see then how the time-symmetric and the time-antisymmetric fluctuations get coupled, and we discuss the validity of

*Electronic address: christian.maes@fys.kuleuven.be

$\dagger$ Electronic address: netocny@fzu.cz 
some entropy production principles. The mathematics involved is the standard Itô/Stratonovich stochastic calculus complemented with the path-integral formalism; for convenience we give a brief review in the appendix together with some additional references.

\section{MODEL AND MAIN RESULTS}

We start with an example of a one-dimensional overdamped Langevin dynamics driven from equilibrium by a nongradient force. This is further generalized to a larger class of diffusions, and in arbitrary dimension.

\section{A. Basic example: diffusion on a circle}

We consider a particle undergoing an overdamped motion on the circle with unit length. This means that we ignore inertial effects, so that forces will be proportional to velocities rather than to acceleration. The particle moves under the influence of a stochastic force (noise), because we imagine the system to be connected to an environment at inverse temperature $\beta$. Added to that, there are also deterministic forces: a periodic potential landscape $U(x)$, and a periodic (nongradient) force $F(x)$, which will drive the system out of equilibrium. Note that on the circle every function $F$ is periodic, but is not in general a derivative of a (periodic) function (for example if $F>0$ everywhere). With some (possibly inhomogeneous) mobility $\chi(x)>0$, this is modeled by the Itô-stochastic dynamics (see Appendix A):

$$
\mathrm{d} x_{t}=\chi\left(x_{t}\right)\left[F\left(x_{t}\right)-U^{\prime}\left(x_{t}\right)\right] \mathrm{d} t+D^{\prime}\left(x_{t}\right) \mathrm{d} t+\sqrt{2 D\left(x_{t}\right)} \mathrm{d} B_{t}
$$

where $\mathrm{d} B_{t}$ is standard Gaussian white noise, so that the process is Markov. The prime as superscript is a shorthand for the spatial derivative. The diffusion coefficient is $D=\chi / \beta$, in agreement with the condition of local detailed balance. The drift counter-term proportional to $D^{\prime}$ then ensures that the case $F=0$ is an equilibrium dynamics, see below. For the following we assume that this stochastic process (1) always relaxes to a unique stationary distribution which mathematically amounts here to smoothness conditions on $F, U$ and $\chi$, see e.g. in [17].

The corresponding Fokker-Planck equation for the time-dependent probability density $\mu_{t}$ is

$$
\frac{\partial \mu_{t}(x)}{\partial t}+j_{\mu_{t}}^{\prime}(x)=0, \quad j_{\mu}=\chi \mu\left(F-U^{\prime}\right)-D \mu^{\prime}
$$

where $j_{\mu}$ is the probability current as the sum of a drift and a diffusion component.

The stationary density, called $\rho$ in the sequel, solves the stationary equation $j_{\rho}^{\prime}=0$, i.e.,

$$
\chi \rho\left(F-U^{\prime}\right)-D \rho^{\prime}=j_{\rho}
$$

is a constant. For $F=0$, equation (3) has the solution

$$
\rho(x)=\frac{1}{Z} e^{-\beta U(x)}, \quad Z=\int_{0}^{1} e^{-\beta U} \mathrm{~d} x
$$

and the corresponding stationary current is $j_{\rho}=0$; this is a detailed balanced dynamics with $\rho$ the equilibrium density. When adding a nongradient driving force, $\int_{0}^{1} F \mathrm{~d} x \neq 0$, the stationary density obtains the form

$$
\rho(x)=\frac{1}{\mathcal{Z}} \int_{0}^{1} \frac{e^{\beta W(y, x)}}{D(y)} \mathrm{d} y, \quad \mathcal{Z}=\int_{0}^{1} \int_{0}^{1} \frac{e^{\beta W(y, x)}}{D(y)} \mathrm{d} y \mathrm{~d} x
$$

where

$$
W(y, x)=U(y)-U(x)+ \begin{cases}\int_{y}^{x} F \mathrm{~d} z & \text { for } y \leq x \\ \int_{y}^{1} F \mathrm{~d} z+\int_{0}^{x} F \mathrm{~d} z & \text { for } y>x\end{cases}
$$

is the work performed by the applied forces along the positively oriented path $y \rightarrow x$. In this model the stationary current can be computed by dividing the stationary equation (3) by $\rho \chi$ and by integration over the circle:

$$
j_{\rho}=\frac{\bar{W}}{\int_{0}^{1}(\rho \chi)^{-1} \mathrm{~d} x}
$$

where $\bar{W}=\int_{0}^{1} F \mathrm{~d} x$ is the work carried over a completed cycle. The non-zero value of this stationary current indicates that time-reversibility is broken. In the simplest nonequilibrium setting when $U=0$ and $F, \chi>0$ are some constants, the steady state has the uniform density $\rho(x)=1$ and the current is $j_{\rho}=\chi F$. 


\section{B. General model}

As a generalization of the driven diffusion on a circle, we consider a class of $d$-dimensional inhomogeneous diffusions introduced by the following equation, which has to be interpreted in the Itô way (see Appendix A):

$$
\mathrm{d} x_{t}=\left\{\chi\left(x_{t}\right)\left[F\left(x_{t}\right)-\nabla U\left(x_{t}\right)\right]+\nabla \cdot D\left(x_{t}\right)\right\} \mathrm{d} t+\sqrt{2 D\left(x_{t}\right)} \mathrm{d} B_{t}
$$

The mobility $\chi(x)$ is now a strictly positive (symmetric) $d \times d$-matrix which is related to the diffusion matrix $D(x)$ as $\chi(x)=\beta D(x)$ with some fixed homogeneous inverse temperature $\beta>0$, and the $d$-dimensional vector $\mathrm{d} B_{t}$ has independent standard Gaussian white noise components. The notation $\nabla \cdot D$ stands for the vector with components $\sum_{\gamma} \partial_{\gamma} D_{\gamma \alpha}$, i.e., with the derivative acting on the left indices of a matrix. From now on we also use the dot to denote scalar product, e.g., $F \cdot G=\sum_{\gamma} F_{\gamma} G_{\gamma}$, whereas no dot is used for a matrix acting on a vector, e.g., $(\chi F)_{\alpha}=\sum_{\gamma} \chi_{\alpha \gamma} F_{\gamma}$.

We restrict ourselves to two types of boundary conditions:

(1) periodic - the particle moves on the unit torus $[0,1)^{d}$ and the fields $U, F$, and $\chi$ are smooth functions on the torus; (2) decay at infinity - the potential $U$ grows fast enough at infinity so that the particle is essentially confined to a bounded region, i.e., the density and its derivative vanish at infinity.

Under either of the above boundary conditions we can simply ignore boundary terms when performing integrations by parts. The particles are essentially confined in their configuration space.

The probability density $\mu_{t}$ evolves according to the Fokker-Planck equation associated with (8):

$$
\frac{\partial \mu_{t}}{\partial t}+\nabla \cdot j_{\mu_{t}}=0, \quad j_{\mu}=\chi \mu(F-\nabla U)-D \nabla \mu
$$

where again $\chi=\beta D$. The stationary condition reads $\nabla \cdot j_{\rho}=0$; in contrast to the previous one-dimensional example the stationary current in general becomes inhomogeneous here. Equation (9) can be interpreted as giving the evolution of the density profile $\mu_{t}$ of a macroscopic amount of independent particles each moving according to (8): at times $t \geq 0$ and for all observables $f$,

$$
\left\langle f\left(x_{t}\right)\right\rangle_{\mu_{0}}=\int f(x) \mu_{t}(x) \mathrm{d} x
$$

where $\langle\cdot\rangle_{\mu_{0}}$ is the average over the path-space distribution started at $\mu_{0}$.

Moreover, the $j_{\mu}$ then also gives the expected profile of the 'real' particle current at given density $\mu$ : again under the diffusion process started from density $\mu_{0}$,

$$
\left\langle\int_{0}^{T} f\left(x_{t}\right) \circ \mathrm{d} x_{t}\right\rangle_{\mu_{0}}=\int_{0}^{T} \mathrm{~d} t \int f(x) j_{\mu_{t}}(x) \mathrm{d} x
$$

which involves the Stratonovich-stochastic integral; see (A8) in Appendix A for its derivation. Taking formally $f(\cdot)=\delta(\cdot-x)$, we indeed recognize $\mu_{t}$ and $j_{\mu_{t}}$ as the time-dependent (or transient) local density, respectively the local current for that density.

\section{Questions and first answers}

Since the Langevin dynamics (10) or (8) is assumed to have a unique stationary distribution, the stationary density $\rho$ can be measured as the most typical time-average of the occupation over a large time interval. Any different measurement outcome, specified by some density $\mu$, is well possible but it is a rare event with an increasingly small probability to be observed as the duration $T \uparrow+\infty$. The large deviation theory, [18, 19], tells us that the generic asymptotic law for such fluctuations is an exponential decay, $\boldsymbol{P}_{T}(\mu) \sim e^{-T I(\mu)}$ with some rate function $I(\mu)$. Here comes a first question:

QA: How can we find the rate function $I(\mu)$ and what is its thermodynamic meaning?

It turns out that $I(\mu)$ can be expressed in terms of the traffic measuring the dynamical activity in the system, and being introduced as a time-symmetric counterpart to the entropy production. We also give a relation between the traffic and the entropy production which appears to be general for diffusion processes.

A very similar question can also be asked about the current statistics, with rate function $I(j)$, but the study of $I(\mu)$ is quite new. 
QB: What are the fluctuations of the time-averaged current around its most probable value $j_{\rho}$, and how are they correlated with the fluctuations of the occupation times? In particular, what are the joint fluctuations for both time-symmetric and time-antisymmetric observables?

To answer these questions, we give an explicit form of the dynamical fluctuation functional $I(\mu, j)$ such that $\boldsymbol{P}(\mu, j) \sim e^{-T I(\mu, j)}$ is the joint probability to see both $\mu$ as the statistics of occupations times and $j$ as the time-averaged current. The functional $I(\mu, j)$ has a general canonical structure and it is given in terms of the entropy production and the traffic. Starting from this basic functional other fluctuation formulas can be obtained by the contraction principle familiar from large deviation theory. For example, one recovers $I(\mu)$ by minimizing $I(\mu, j)$ over all admissible currents $j$.

The above questions and the methods that we take below are not entirely original. They have appeared in the mathematical literature in a systematic way since the theory of large deviations was introduced in the framework of Markov diffusion processes, see [18, 19, 20]. The relevance to physics and to statistical mechanics in particular is obvious, but the thermodynamic interpretation of the resulting dynamical fluctuation functionals has not been systematically investigated. A first study can be found in [16]. Our paper will add to that, starting from the next section. There have of course been many other studies of dynamical fluctuation theory in the literature. We mention in particular the works of Derrida and Bodineau, see [8, 9] and of Bertini et al, e.g. in [3, 7]. As we are dealing here with diffusion processes, our approach is especially similar to what one is doing for a macrostatistical theory, where the hydrodynamic fluctuations can be viewed as solution of some infinite-dimensional diffusion process. Possible differences with the existing work are first of all that the problems related to the diffusion-approximation or to a hydrodynamic rescaling do not enter in our work. We just start from a finite-dimensional diffusion process as such, in the same way as we could start from a Markov jump process, boundary or bulk driven, and without extra rescaling. In other words, we prefer to split the problem of hydrodynamical scaling with possible diffusion approximation from the problem of studying the dynamical fluctuations. Secondly, we are concerned here directly with the stationary fluctuations, without passing via the transient regime. Thirdly, as soon will become clear, we like to emphasize a (thermodynamic) canonical structure in the joint fluctuations of density and current. In order to achieve that, it is natural to introduce the novel concept of traffic (as in the next section) whose thermodynamic interpretation remains to be fully elucidated. Our three main results are in Sections IVB, IVD and VA.

\section{THERMODYNAMIC CONSIDERATIONS}

In this section we introduce the two main players in the presented fluctuation theory: the entropy production and the traffic. While the former notion is rather standard, the latter appears to be new.

\section{A. Entropy production}

The entropy production is the change of entropy in the world between two moments in a process. The very notion of entropy is however controversial when trying to extend it to nonequilibrium situations. The problem is mainly that we do not have a good understanding of the relevant quantities or of the macroscopic variables that characterize the nonequilibrium state and its evolution. Nevertheless we can try to work with the notion of entropy production as it has come to us from considerations of heterogeneous or of local equilibrium. There are two basic contributions to that entropy production.

The entropy of a closed macroscopic system is defined via counting microstates, which at the same time gives both the static fluctuation theory with the entropy as a fluctuation functional and a second law inequality, 21]. For a mesoscopic system this cannot be applied as such. However, one can think of an ensemble of $N \rightarrow \infty$ independent copies. A density $\mu$ then becomes a macro-observable telling us the relative occupation of the space, and the associated counting entropy equals the relative entropy (with respect to the flat distribution):

$$
s(\mu)=-\int \mu(x) \log \mu(x) \mathrm{d} x
$$

By construction it is the (static) fluctuation functional in the probability law for observing the empirical density $\mu$ when sampling the particles from the flat distribution. For $F=\nabla U=0$ (i.e. for a thermodynamically closed system) the entropy rate satisfies $\mathrm{d} s / \mathrm{d} t \geq 0$ along the solution of the Fokker-Planck equation.

An open system dissipates heat that results in an extra entropy production in the environment. The rate $\mathcal{Q}(\mu)$ of mean heat dissipation comes from the work performed by the nongradient force and from the change of the energy of 
the system:

$$
\begin{aligned}
\mathcal{Q}(\mu) & =\int F \cdot j_{\mu} \mathrm{d} x-\frac{\mathrm{d}}{\mathrm{d} t} \int U \mu \mathrm{d} x \\
& =\int(F-\nabla U) \cdot j_{\mu} \mathrm{d} x+\int \nabla \cdot\left(U j_{\mu}\right) \mathrm{d} x
\end{aligned}
$$

where we have used the Fokker-Planck equation (9). Under either of the two boundary conditions considered in this paper (i.e. periodic or decaying at infinity, see Section IB $)$, the last integral equals zero. That argument will often come back in what follows.

The environment is a heat reservoir at inverse temperature $\beta$ that remains itself at equilibrium during the whole process (the weak coupling assumption). Hence, the mean entropy flux equals $\beta \mathcal{Q}(\mu)$ and the total entropy production rate reads

$$
\begin{aligned}
\sigma(\mu) & =\beta \mathcal{Q}(\mu)+\frac{\mathrm{d} s}{\mathrm{~d} t}(\mu) \\
& =\int(\beta F-\beta \nabla U-\nabla \log \mu) \cdot j_{\mu} \mathrm{d} x \\
& =\int j_{\mu} \cdot(\mu D)^{-1} j_{\mu} \mathrm{d} x
\end{aligned}
$$

where we have used again the Fokker-Planck equation (9) and the boundary conditions. In the language of irreversible thermodynamics, $\beta F-\nabla \log \left(\mu e^{\beta U}\right)$ is a generalized thermodynamic force conjugated to the current $j_{\mu}$. Clearly, $\sigma(\mu) \geq 0$ in agreement with the second law, proving the thermodynamic consistency of our diffusion model.

\section{B. Dynamical entropy and traffic}

The diffusion as defined by the stochastic equation (8) is a Markov process. Its randomness can be characterized by the dynamical entropy which is a dynamical variant of the static entropy (12) obtained by replacing the density $\mu$ with the path-space distribution of the process. Such a construction is often useful in the theory of dynamical and stochastic systems; here we review how it is linked to the thermodynamic entropy production defined in the previous section and what other information it provides.

We start by writing the density of the path-distribution $\boldsymbol{P}_{\mu_{0}}$ of the process with respect to a suitable reference process $\boldsymbol{P}^{0}$, for which we take the one corresponding to $F=\nabla U=0$ (see Appendix B):

$$
\mathrm{d} \boldsymbol{P}_{\mu_{0}}(\omega)=\mu_{0}\left(x_{0}\right) e^{-\int_{0}^{T} \mathcal{L}^{+}\left(x_{t}\right) \mathrm{d} t-\int_{0}^{T} \mathcal{L}^{-}\left(x_{t}, \mathrm{~d} x_{t}\right)} \mathrm{d} \boldsymbol{P}^{0}(\omega)
$$

where $\omega=\left(x_{t}\right)_{t=0}^{T}$ is a trajectory and $\mu_{0}$ is an initial density. The weight in the exponential is split in a time-symmetric part

$$
\mathcal{L}^{+}(x)=\frac{\beta^{2}}{4}[(F-\nabla U) \cdot D(F-\nabla U)]+\frac{\beta}{2} \nabla \cdot[D(F-\nabla U)]
$$

and a time-antisymmetric part

$$
\mathcal{L}^{-}(x, \mathrm{~d} x)=-\frac{\beta}{2}(F-\nabla U) \circ \mathrm{d} x
$$

The dynamical entropy is the relative entropy of the path-distribution $\boldsymbol{P}_{\mu}$ with respect to the reference $\boldsymbol{P}^{0}$, over some fixed time interval $[0, T]$ :

$$
\mathcal{D}\left(\mu_{0}\right)=\left\langle\log \frac{\mathrm{d} P_{\mu_{0}}}{\mathrm{~d} P^{0}}\right\rangle_{\mu_{0}}=-s\left(\mu_{0}\right)-\left\langle\int_{0}^{T} \mathcal{L}^{+}\left(x_{t}\right) \mathrm{d} t\right\rangle_{\mu_{0}}-\left\langle\int_{0}^{T} \mathcal{L}^{-}\left(x_{t}, \mathrm{~d} x_{t}\right)\right\rangle_{\mu_{0}}
$$

The time-antisymmetric contribution to the dynamical entropy equals, applying the identity (11),

$$
-\left\langle\int_{0}^{T} \mathcal{L}^{-}\left(x_{t}, \mathrm{~d} x_{t}\right)\right\rangle_{\mu_{0}}=\frac{\beta}{2} \int_{0}^{T} \mathrm{~d} t \int(F-\nabla U) j_{\mu_{t}} \mathrm{~d} x=\frac{\beta}{2} \int_{0}^{T} \mathcal{Q}\left(\mu_{t}\right) \mathrm{d} t
$$


Hence, it is given in terms of the entropy flux. This is an instance of a fairly general observation that lies behind a famous fluctuation symmetry of the entropy production, 22, 23, 24].

The time-symmetric contribution is computed analogously via (10) to obtain

$$
\left\langle\int_{0}^{T} \mathcal{L}^{+}\left(x_{t}\right) \mathrm{d} t\right\rangle_{\mu_{0}}=\int_{0}^{T} \mathcal{T}\left(\mu_{t}\right) \mathrm{d} t
$$

where we have introduced a new quantity $\mathcal{T}(\mu)$, called traffic, which equals

$$
\mathcal{T}(\mu)=\frac{\beta^{2}}{4} \int \mu(F-\nabla U) \cdot D(F-\nabla U) \mathrm{d} x+\frac{\beta}{2} \int \mu \nabla \cdot[D(F-\nabla U)] \mathrm{d} x
$$

By construction, the traffic is that part of the dynamical entropy which originates from the time-symmetric fluctuations. Finally, we arrive at the next two equivalent expressions for the dynamical entropy (18):

$$
\begin{aligned}
\mathcal{D}\left(\mu_{0}\right) & =-s\left(\mu_{0}\right)-\int_{0}^{T} \mathcal{T}\left(\mu_{t}\right) \mathrm{d} t+\frac{\beta}{2} \int_{0}^{T} \mathcal{Q}\left(\mu_{t}\right) \mathrm{d} t \\
& =-\frac{1}{2}\left[s\left(\mu_{0}\right)+s\left(\mu_{T}\right)+2 \int_{0}^{T} \mathcal{T}\left(\mu_{t}\right) \mathrm{d} t-\int_{0}^{T} \sigma\left(\mu_{t}\right) \mathrm{d} t\right]
\end{aligned}
$$

where we have inserted (14).

\section{Traffic versus entropy production}

A remarkable and important feature of diffusion systems, not valid in general beyond the diffusion approximation, [16], is that the traffic (21) and the entropy production (14) are not independent of each other. Indeed, one checks the relation

$$
\mathcal{T}(\mu)=\frac{\sigma(\mu)}{4}+\int \frac{\nabla \mu \cdot D \nabla \mu}{4 \mu} \mathrm{d} x
$$

in which the last term only depends on the distribution $\mu$ and not on the imposed potential $U$ nor on the driving $F$. As we will see in the next section, differences in the traffic and in the entropy production when varying $U$ and $F$ determine the asymptotics of dynamical fluctuations. Hence, relation (23) brings about a simplification in the structure of fluctuations that is characteristic and restricted to diffusions. We believe that this also indicates that a more general nonequilibrium theory should also reach beyond the Langevin or diffusion approximation.

\section{JOINT OCCUPATION-CURRENT STATISTICS}

According to Einstein's fluctuation theory, the entropy and derived quantities govern the structure of static fluctuations. In this section we explain how the time-symmetric and time-antisymmetric components of the dynamical entropy play an essential role in dynamical fluctuation theory.

\section{A. Definitions}

A basic and time-symmetric dynamical observable is the empirical density of the occupation times defined as the fraction of time spent in the neighborhood of every point $x$, over a fixed time interval $T$ :

$$
\bar{\mu}_{T}(x)=\frac{1}{T} \int_{0}^{T} \delta\left(x_{t}-x\right) \mathrm{d} t
$$

This is a path-dependent observable as it varies over the paths $\omega=\left(x_{t}\right)_{t=0}^{T}$. For the large time asymptotics $T \rightarrow \infty$, we have $\bar{\mu}_{T} \rightarrow \rho$ almost surely and independently of the initial condition. We will be concerned with physically determining the fluctuation functional $I(\mu)$ that enters the large deviation law

$$
\boldsymbol{P}\left(\bar{\mu}_{T}=\mu\right) \sim e^{-T I(\mu)}
$$


That has to be understood as an asymptotic formula that becomes an equality after taking the logarithm and dividing by $T \rightarrow \infty$ in both left- and right-hand sides; see [19, 25] for a more precise mathematical formulation.

The time-antisymmetric observable of special relevance is the empirical current, formally defined as

$$
\bar{j}_{T}(x)=\frac{1}{T} \int_{0}^{T} \delta\left(x_{t}-x\right) \circ \mathrm{d} x_{t}
$$

It depends again on the (random) path $\omega=\left(x_{t}\right)_{t=0}^{T}$ and it measures the time-averaged current while in $x$, as in (11). Its steady average equals $j_{\rho}$; moreover, $\bar{j}_{T} \rightarrow j_{\rho}$ for $T \rightarrow \infty$ almost surely. The rate function $I(j)$ is defined analogously to (25), but for the probability $\boldsymbol{P}\left(\bar{j}_{T}=j\right)$.

Both $I(\mu)$ and $I(j)$ can be obtained in principle from the joint fluctuations. The large time asymptotics of the joint fluctuations of $\bar{\mu}_{T}$ and $\bar{j}_{T}$ is described by the fluctuation functional $I(\mu, j)$ such that

$$
\boldsymbol{P}\left(\bar{\mu}_{T}=\mu ; \bar{j}_{T}=j\right) \sim e^{-T I(\mu, j)}
$$

always logarithmically when $T \rightarrow \infty$. A first observation is that $I(\mu, j)=\infty=I(j)$ whenever $j$ is not stationary, i.e. for $\nabla \cdot j \neq 0$. Indeed, for any smooth bounded function $Y$ one has

$$
\int Y \nabla \cdot \bar{j}_{T} \mathrm{~d} x=-\frac{1}{T} \int_{0}^{T} \nabla Y\left(x_{t}\right) \circ \mathrm{d} x_{t}=-\frac{1}{T}\left[Y\left(x_{T}\right)-Y\left(x_{0}\right)\right] \rightarrow 0
$$

and hence, in a distributional sense, $\nabla \cdot \bar{j}_{T} \rightarrow 0$ for $T \rightarrow \infty$ along any particle trajectory, which proves the above statement. That is why from now on we always assume that $\nabla \cdot j=0$, unless otherwise specified.

\section{B. Result 1: traffic and entropy production determining the joint fluctuations}

To compute $I(\mu, j)$ we use a standard large deviation technique, sometimes referred to as Cramér tilting, see e.g. [19, 20]. We modify the driving of the original dynamics changing $F$ into some $G$ and we take care that this new and modified dynamics is chosen so that $\mu$ and $j$ become both stationary (and hence are typical as large time averages). The potential $U$ remains untouched. The more formal argument goes as follows.

Let $\boldsymbol{P}_{F}$ and $\boldsymbol{P}_{G}$ be the stationary path-space distributions for driving $F$ respectively $G$, both processes started at their respective stationary distribution. We refer to Appendix B for that notion of path-space distribution. We must compute the probability $\boldsymbol{P}_{F}[A]$ of the event $A$ containing all the paths $\omega$ over time $T$ such that $\bar{\mu}_{T}=\mu$ and $\bar{j}_{T}=j$, see (27):

$$
\boldsymbol{P}_{F}[A]=\int \mathrm{d} \boldsymbol{P}_{G}(\omega) \frac{\mathrm{d} \boldsymbol{P}_{F}}{\mathrm{~d} \boldsymbol{P}_{G}}(\omega) \chi\left[\bar{\mu}_{T}=\mu ; \bar{j}_{T}=j\right]
$$

The density of path-space distribution $\boldsymbol{P}_{F}$ with respect to path-space distribution $\boldsymbol{P}_{G}$ can be written out explicitly starting from (B3): we find

$$
\frac{\mathrm{d} \boldsymbol{P}_{F}}{\mathrm{~d} \boldsymbol{P}_{G}}(\omega)=\frac{\rho\left(x_{0}\right)}{\mu\left(x_{0}\right)} \exp \left[\int_{0}^{T} \mathrm{~d} t\left(\mathcal{L}_{G}^{+}-\mathcal{L}_{F}^{+}+\mathcal{L}_{G}^{-}-\mathcal{L}_{F}^{-}\right)\right]
$$

where we have made use of definitions (16) and (17). The point is now that this can be fully expressed as a function of the path-dependent occupation and current fractions; in other words, when indeed $A$ occurs, that is, when $\bar{\mu}_{T}=\mu$ and $\bar{j}_{T}=j$, then

$$
\begin{aligned}
\int_{0}^{T} \mathcal{L}_{G}^{+}\left(x_{t}\right) \mathrm{d} t & =T \int \mu(x) \mathcal{L}_{G}^{+}(x) \mathrm{d} x=T \mathcal{T}_{G}(\mu) \\
\int_{0}^{T} \mathcal{L}_{G}^{-}\left(x_{t}, \mathrm{~d} x_{t}\right) & =-\frac{\beta}{2} \int \mathrm{d} x(G-\nabla U)(x) \cdot \int_{0}^{T} \delta\left(x_{t}-x\right) \circ \mathrm{d} x_{t} \\
& =-\frac{\beta T}{2} \int G \cdot j \mathrm{~d} x
\end{aligned}
$$


As a consequence (29) simplifies: the density between the path-space distributions comes out of the path-integral and since by construction, for $T \rightarrow \infty, \boldsymbol{P}_{G}[A] \rightarrow 1$, we get (27) in the form

$$
I(\mu, j)=\mathcal{T}_{F}(\mu)-\mathcal{T}_{G}(\mu)+\frac{\beta}{2} \int(G-F) \cdot j \mathrm{~d} x
$$

That is to be read as the sum of an excess instantaneous traffic given density $\mu$ alone, and an excess work (or, equivalently, entropy flux) given stationary current $j$ alone. The excess is being understood in the sense of the above modification, with $G$ producing the current $j$, i.e., $j=\chi \mu(G-\nabla U)-D \nabla \mu$, see (9), or

$$
\beta G=(\mu D)^{-1} j+\nabla \log \left(\mu e^{\beta U}\right)
$$

Since, by (23), excess entropy production equals excess traffic (for our diffusion processes), we can equivalently write (32) as

$$
I(\mu, j)=\frac{\sigma(\mu)-\sigma_{G}(\mu)}{4}+\frac{\beta}{2} \int(G-F) \cdot j \mathrm{~d} x
$$

Upon substituting (33) into (34) and using (14), the fluctuation functional can also be written in the following explicitly positive form:

$$
\begin{aligned}
I(\mu, j) & =\frac{\sigma(\mu)-\sigma_{G}(\mu)}{4}+\frac{1}{2} \int(\mu D)^{-1}\left(j-j_{\mu}\right) \cdot j \mathrm{~d} x \\
& =\frac{1}{4} \int\left(j-j_{\mu}\right) \cdot(\mu D)^{-1}\left(j-j_{\mu}\right) \mathrm{d} x
\end{aligned}
$$

(On the assumption $\nabla \cdot j=0$; remember that $I(\mu, j)=\infty$ otherwise.) This last formula resembles the Gaussian-like expressions for the current distribution, typical for hydrodynamic fluctuations of the diffusion-type. Such expressions are omnipresent in the works of e.g. [7, 8]. Although the quadratic integrand in (35) resembles the (generalized Onsager-Machlup) Lagrangian for macroscopic fluctuations in the hydrodynamic limit, we have no spatial/temporal rescaling here. We have started from a mesoscopic system as described by a diffusion equation and the only large parameter is the time span $T$. What we stress here with respect to other work on dynamical large deviations, becomes visible from the formulæ(32) and (34) and has as such nothing to do with hydrodynamic rescaling or with macrostatistics. It concerns the thermodynamic interpretation of the fluctuation functional $I(\mu, j)$ for our mesoscopic system: how it is shaped from quantities like traffic, work and entropy production, and providing full account of the steady dynamical fluctuations in both the time-symmetric and the time-antisymmetric sectors.

\section{Steady state fluctuation symmetry}

As proof of internal consistency we check the fluctuation symmetry, cf. [5, [6, 24].

As a corollary of (35), one has

$$
I(\mu,-j)-I(\mu, j)=\int j \cdot(\mu D)^{-1} j_{\mu} \mathrm{d} x=\mathcal{S}(j)
$$

where

$$
\begin{aligned}
\mathcal{S}(j) & =\beta \int F \cdot j \mathrm{~d} x \\
& =\frac{\beta}{T} \int_{0}^{T} F\left(x_{t}\right) \circ \mathrm{d} x_{t} \quad\left(\text { when } \bar{j}_{T}=j\right)
\end{aligned}
$$

is $\beta$ times the time-averaged power of the nongradient forces under the condition that $\bar{j}_{T}=j$ is the time-averaged current. Due to the stationary condition $(\nabla \cdot j=0)$ it also coincides with the entropy flux.

\section{Result 2: canonical structure of the joint fluctuations}

A useful feature of equilibrium statistical thermodynamics is that the fluctuation functions can in a simple and general way be given as differences of thermodynamic potentials that generate the relevant order parameters. As 
suggested already by formula (32), a similar structure also emerges for the dynamical fluctuations. In order to make that manifest we should follow the exact dependence on the driving $F$, and we therefore indicate that dependence here explicitly. In that way, we have an immediate rewriting of (32):

$$
I_{F}(\mu, j)=I_{0}(\mu, j)-\frac{\mathcal{S}_{F}(j)}{2}+\mathcal{T}_{F}(\mu)-\mathcal{T}_{0}(\mu)
$$

where $I_{0}(\mu, j)$ is the fluctuation functional for the reference equilibrium process with no driving $(F=0)$, traffic was defined in (21) and the entropy flux follows from (37). The canonical structure of that fluctuation functional arises because the last difference, the excess traffic

$$
H(\mu, F)=2\left[\mathcal{T}_{F}(\mu)-\mathcal{T}_{0}(\mu)\right]
$$

is a potential for the current in the sense that

$$
\frac{\delta H(\mu, F)}{\delta F(x)}=2 \frac{\delta \mathcal{T}_{F}(\mu)}{\delta F(x)}=\beta j_{\mu}^{F}(x)
$$

Its Legendre transform is

$$
G(\mu, j)=\sup _{F}\left[\mathcal{S}_{F}(j)-H(\mu, F)\right]
$$

with associated

$$
\frac{\delta G}{\delta j(x)}\left(\mu, j_{\mu}^{F}\right)=\beta F(x)
$$

so that force and current $(F, j)$ make a canonical pair. It can be checked immediately that

$$
G(\mu, j)=\frac{1}{2} \int\left(j-j_{\mu}^{0}\right) \cdot(\mu D)^{-1}\left(j-j_{\mu}^{0}\right) \mathrm{d} x
$$

which coincides with $2 I_{0}(\mu, j)$ whenever $\nabla \cdot j=0$. Using that extended functional to replace $I_{0}(\mu, j)$ in (38), we get

$$
I_{F}(\mu, j)=\frac{1}{2}\left[G(\mu, j)-\mathcal{S}_{F}(j)+H(\mu, F)\right]
$$

for all densities $\mu$ and stationary currents $j$.

We can still rewrite (42) in the form

$$
4 I_{F}(\mu, j)=\sup _{F}\left\{2 \mathcal{S}_{F}(j)-\sigma_{F}(\mu)\right\}-2 \mathcal{S}_{F}(j)+\sigma_{F}(\mu)
$$

fully in terms of entropic quantities, due to (23).

A similar canonical structure, cf. (39) and (40), has been established already before in the framework of jump processes at least on a sufficiently fine-grained scale of description, see [16]. One can therefore conclude that the structure of (42) is also canonical in the sense of being generally valid for a very large class of dynamics.

\section{E. Small fluctuations}

We look here at the Gaussian approximation in a dynamics far from equilibrium. Later we will also make the driving $F$ small, to be close to equilibrium.

As is clear from (35), current and occupations are coupled. It is because of this coupling that contractions of $I(\mu, j)$ to $I(\mu)$ and to $I(j)$ become rather complicated. Even for small fluctuations this coupling remains: take $\mu=\rho\left(1+\epsilon \mu_{1}\right)$ and $j=j_{\rho}+\epsilon j_{1}$, with $\epsilon$ a small parameter. Because $j-j_{\mu}$ is then $O(\epsilon)$, the fluctuation functional is $O\left(\epsilon^{2}\right)$ :

$$
\begin{array}{rl}
I(\mu, j)=\frac{\epsilon^{2}}{4} \int \mathrm{d} & x\left[j_{1} \cdot(\rho D)^{-1} j_{1}+\mu_{1}^{2} j_{\rho} \cdot(\rho D)^{-1} j_{\rho}\right. \\
+ & \left.\nabla \mu_{1} \cdot \rho D \nabla \mu_{1}-2 \mu_{1} j_{1} \cdot(\rho D)^{-1} j_{\rho}\right]+O\left(\epsilon^{3}\right)
\end{array}
$$


The last term in this approximation gives the coupling between occupation and current fluctuations. It is proportional to the stationary current, which is non-zero away from equilibrium. It is only when we take a dynamics close to equilibrium, i.e. $F=\epsilon F_{1}$, that the fluctuations decouple. In this approximation we have that $j_{\rho}=O(\epsilon)$, and thus, near equilibrium,

$$
I(\mu, j)=\frac{\epsilon^{2}}{4} \int \mathrm{d} x\left[j_{1} \cdot(\rho D)^{-1} j_{1}+\nabla \mu_{1} \cdot \rho D \nabla \mu_{1}\right]+O\left(\epsilon^{3}\right)
$$

with, to leading order, a complete decoupling between the time-symmetric and the time-antisymmetric sectors.

\section{CONTRACTIONS}

Now that we have a fluctuation functional for both symmetric and antisymmetric variables, we can compute the statistics of empirical averages of arbitrary physical quantities. In particular, we can try to find the fluctuation functionals for density $I(\mu)$ and and for current $I(j)$ separately. The technique to do that is called contraction as we go so to speak to a more contracted description. To start we look at the fluctuations of the density (alone).

\section{A. Result 3: occupation statistics}

Look back at the definition (25). As $I(\mu)=\inf _{j} I(\mu, j)$, we have to compute the minimizing current $j$ for any given density $\mu$. Since the minimization is constrained via the stationary condition $\nabla \cdot j=0$, we get the equation

$$
j=\chi \mu[F-\nabla \cdot(U+\psi)]-D \nabla \mu
$$

where $\psi$ is a Lagrange multiplier (function of $x$ ). Not surprisingly, we see that the minimizer is the stationary current for a modified dynamics that makes $\mu$ stationary. This modified dynamics is achieved here by replacing the imposed potential $U$ with a modified one, called $V=U+\psi$ (for some $\psi$ ). We therefore call the minimizing current in (46) $j_{\mu}^{V}$, and the fluctuation functional becomes:

$$
I(\mu)=\frac{1}{4} \int\left(j_{\mu}^{V}-j_{\mu}\right) \cdot(\mu D)^{-1}\left(j_{\mu}^{V}-j_{\mu}\right) \mathrm{d} x
$$

For some explicit examples of solutions to (46), see furtherdown, in equations (50)-(56).

The fluctuation functional $I(\mu)$ obtains other equivalent forms by following a road backward from the one that led us before to (35):

$$
I(\mu)=\mathcal{T}(\mu)-\mathcal{T}_{V}(\mu)=\frac{\sigma(\mu)-\sigma_{V}(\mu)}{4}
$$

where the second equality follows again from (23). In this way we have recognized the excess traffic (or here also: the excess entropy production) as governing the large time statistics of the occupation times. That constitutes our third main result: the fluctuation functional $I(\mu)$ exactly equals one quarter of a difference in entropy production rates when having density $\mu$, these rates being computed respectively for the original dynamics and for a modified dynamics that makes $\mu$ stationary.

In formulæ (47) and (48) the potential $V$ has to be determined from $\mu$ by solving the inverse stationary problem (46). We now give two classes of examples where this $V$ and hence $I(\mu)$ can be made explicit.

Diffusion on the circle. For the one-dimensional example of Section II A the inverse stationary problem (46) allows for an explicit solution. The current $j_{\mu}^{V}$ is immediately read off the formula (7),

$$
j_{\mu}^{V}=\frac{\beta \bar{W}}{\int_{0}^{1}(\mu D)^{-1} \mathrm{~d} x}, \quad \bar{W}=\int_{0}^{1} F \mathrm{~d} x
$$

and the potential $V$ obtains the form

$$
V(x)=-\frac{1}{\beta} \log \mu(x)+\int_{0}^{x}\left(F-\frac{j_{\mu}^{V}}{\beta \mu D}\right) \mathrm{d} y
$$


which is a nonlocal functional of the given density $\mu$. The fluctuation functional is explicitly given as

$$
4 I(\mu)=\sigma(\mu)-\frac{\bar{W}^{2}}{\int_{0}^{1}(\mu D)^{-1} \mathrm{~d} x}
$$

for $\mu \neq 0$.

Observe that if $\mu=0$ on some open set $A$ then the rate function equals $I(\mu)=\sigma(\mu) / 4$. (That follows also from the equilibrium form (54) below as the circle gets effectively cut and the dynamics mimics a detailed balance one.) The infimum of $I(\mu)$ over all densities $\mu$ that vanish on $A$ then gives the escape rate from the complement $A^{c}=[0,1) \backslash A$. As a simple example, assume that $U=0$ and let $F$ and $D$ be some constants. In this case the entropy production (14) reads

$$
\sigma(\mu)=\beta^{2} D F^{2}+D \int_{0}^{1} \frac{\mu^{\prime 2}}{\mu} \mathrm{d} x
$$

To compute the escape rate from $A^{c}$ (or, entrance rate to $A$ ) we must take the infimum of (52) over all $\mu$ that vanish on $A$. Setting $A=(0, \delta)$ for some $0<\delta<1$, that infimum is reached for the density $\mu^{*}(x)=\frac{2}{1-\delta} \sin ^{2}\left(\frac{\pi(x-\delta)}{1-\delta}\right), x \in[\delta, 1]$, and the escape rate is

$$
\inf _{\left.\mu\right|_{A}=0} I(\mu)=I\left(\mu^{*}\right)=\frac{\pi^{2} D}{(1-\delta)^{2}}+\frac{\beta^{2} D F^{2}}{4}
$$

Even in equilibrium $(F=0)$ the result is meaningful as it relates the diffusion constant to an escape rate. In the context of dynamical systems, the analysis of the escape rates and of their link to linear transport coefficients was initiated by Dorfman and Gaspard, see [26, 27] and references therein.

Close-to-equilibrium dynamics. Let us start from what can be said in general for equilibrium diffusions. If $F=0$ then equation (46) has the solution $V=-\beta^{-1} \log \mu$, and the corresponding current $j_{\mu}^{V}$ and the entropy production $\sigma_{V}(\mu)$ are both zero. As a result,

$$
I(\mu)=\frac{\sigma(\mu)}{4}
$$

This exact relation between the equilibrium dynamical fluctuations and the entropy production is solely true for diffusion processes. In contrast, for jump processes $\sigma(\mu)$ gives only the leading term in an expansion of $I(\mu)$ around the equilibrium density $\rho \propto e^{-\beta U}$, and the relation (54) obtains corrections when beyond small fluctuations; see [14] for details.

When close to equilibrium, the computation of $I(\mu)$ by contraction is easy: we see from (45) that the second term on its right-hand side is just $I(\mu)$.

In the same approximation of small fluctuations and close-to-equilibrium, the entropy production becomes:

$$
\sigma(\mu)=\int \mathrm{d} x\left[j_{\rho} \cdot(\rho D)^{-1} j_{\rho}+\epsilon^{2} \nabla \mu_{1} \cdot \rho D \nabla \mu_{1}\right]+O\left(\epsilon^{3}\right)
$$

and thus we get

$$
I(\mu)=\frac{\sigma(\mu)-\sigma(\rho)}{4}+O\left(\epsilon^{3}\right)
$$

This reveals to be a special case of a general result, [14], according to which the entropy production governs the occupational statistics in the linear irreversible regime. It provides a fluctuation-based explanation for the minimum entropy production principle introduced by Prigogine to characterize stationarity via an (approximate) variational principle, [28]: the stationary state has minimal (not necessarily zero) entropy production.

\section{B. Current statistics}

The contraction to the current $j$ is also possible. However, up to special examples, there is no explicit solution to the associated variational problem and for general models one has to resort to a perturbative or numerical analysis. In fact, often the calculation starting from the generating function of the current appears more practical than to do 
the contraction starting from $I(\mu, j)$, see $[9,11]$.

We restrict us here to giving the result for a constant drift on the circle and to small fluctuations around equilibrium.

Constantly driven diffusion on the circle. Again we take $U=0$ and $F, \chi$ constants. In this case, from (35) the joined fluctuation functional reads:

$$
I(\mu, j)=\frac{1}{4 D} \int \frac{1}{\mu}\left(j-\beta D F \mu-D \mu^{\prime}\right)^{2} \mathrm{~d} x
$$

and for all $j$, the infimum over $\mu$ is reached at the uniform distribution, so that

$$
I(j)=\frac{(j-\beta D F)^{2}}{4 D}
$$

and hence we see that here the current fluctuations are Gaussian.

Close-to-equilibrium. We have the analogue of the minimum entropy production principle. The starting point is again (45) from which we extract the current fluctuations:

$$
\begin{aligned}
I(j) & =\frac{1}{4} \int\left(j-j_{\rho}\right) \cdot(\rho D)^{-1}\left(j-j_{\rho}\right) \mathrm{d} x+O\left(\epsilon^{3}\right) \\
& =\frac{1}{4}\left[\mathscr{D}\left(j_{\rho}\right)+\mathscr{D}(j)-2 \mathcal{S}(j)\right]+O\left(\epsilon^{3}\right)
\end{aligned}
$$

with $\mathscr{D}(j)=\int j \cdot(\rho D)^{-1} j \mathrm{~d} x$ sometimes called the Onsager dissipation function, and $\mathcal{S}(j)$ is the entropy flux defined in (36). In particular, this leads to a variational characterization of the steady current $j_{\rho}$ which can be written as the following maximum entropy production principle: the $j_{\rho}$ maximizes the entropy flux $\mathcal{S}(j)$ under the two stationary constraints

$$
\text { (1) } \nabla \cdot j=0, \quad(2) \mathscr{D}(j)=\mathcal{S}(j)
$$

The second condition is indeed satisfied at $j=j_{\rho}$ (note also that $\rho$ can with no harm in this order be replaced by the equilibrium density $\rho_{F=0}=e^{-\beta U} / Z$.) Such a variational principle, known as a maximum entropy production principle, is often used in applications and apparently even beyond the linear irreversible regime. As is however clear from (59) from our dynamical fluctuation theory, the validity of the maximum entropy principle is restricted to close-to-equilibrium. Beyond that regime, we must refer to contractions from (32), (42) or even from (44) for generally valid expressions with a general thermodynamic meaning.

\section{Acknowledgments}

K.N. acknowledges the support from the Grant Agency of the Czech Republic (Grant no. 202/07/0404). C.M. benefits from the Belgian Interuniversity Attraction Poles Programme P6/02. B.W. is an aspirant of FWO, Flanders. C.M. and B.W. thank the Institute of Physics of the Academy of Sciences in Prague for its kind hospitality.

\section{APPENDIX A: STOCHASTIC INTEGRALS}

We collect here some necessary albeit formal manipulations from stochastic calculus. We refer to [29] and [17] for further systematics.

Physical quantities, such as work and heat dissipation, depend on the specific trajectory (or path) that the system covers during its evolution. But for diffusions these paths are not differentiable. Therefore integrals like $\int_{0}^{T} f\left(x_{t}\right) \mathrm{d} x_{t}$ cannot be defined in the usual way. It turns out that these integrals, called stochastic integrals, can be interpreted in different ways. Most common are the Itô and Stratonovich interpretations, see e.g. [29].

Itô integral. For the Itô interpretation, the integral domain $[0, T]$ is split up in a set of discrete points $0=t_{0}<$ $t_{1}<\ldots<t_{n}=T$, with $\Delta t_{j}=t_{j}-t_{j-1}$, such that $\Delta t \equiv \max _{j} \Delta t_{j} \rightarrow 0$ for $n \rightarrow \infty$. It is important to note that for diffusions (8) we have that for $\Delta t_{j} \rightarrow 0$,

$$
\left(\Delta x_{j}^{(\alpha)}\right)\left(\Delta x_{j}^{(\beta)}\right)=\left(x_{t_{j}}^{(\alpha)}-x_{t_{j-1}}^{(\alpha)}\right)\left(x_{t_{j}}^{(\beta)}-x_{t_{j-1}}^{(\beta)}\right) \rightarrow 2 D_{\alpha \beta}\left(x_{t_{j}}\right) \Delta t_{j}
$$


where $\alpha$ and $\beta$ denote the components of the vectors and the diffusion matrix. The stochastic integral is then computed as

$$
\int_{0}^{T} f\left(x_{t}\right) \mathrm{d} x_{t}=\lim _{n \rightarrow \infty, \Delta t \rightarrow 0} \sum_{j=1}^{n} f\left(x_{t_{j-1}}\right) \cdot\left(x_{t_{j}}-x_{t_{j-1}}\right)
$$

Note that the function $f$ can be either scalar or vector, and it is evaluated at the left endpoint of the intervals $\left[t_{j-1}, t_{j}\right]$.

For the Itô integral one cannot use the normal rules of integration. Instead it is easily checked from (A1) that for any function $g$,

$$
\int_{0}^{T} \nabla g\left(x_{t}\right) \cdot \mathrm{d} x_{t}=g\left(x_{T}\right)-g\left(x_{0}\right)-\int_{0}^{T}(D \nabla \cdot \nabla g)\left(x_{t}\right) \mathrm{d} t
$$

(The symbol $\cdot$ stands for the scalar product.)

Stratonovich integral. The Stratonovich interpretation differs from the Itô interpretation only in the points of evaluation of the function $f$. In this case $f$ is evaluated in the midpoints of the time intervals:

$$
\begin{aligned}
\int_{0}^{T} f\left(x_{t}\right) \circ \mathrm{d} x_{t} & =\lim _{n \rightarrow \infty, \Delta t \rightarrow 0} \sum_{j=1}^{n} f\left(\frac{x_{t_{j}}+x_{t_{j-1}}}{2}\right) \cdot\left(x_{t_{j}}-x_{t_{j-1}}\right) \\
& =\frac{1}{2} \lim _{n \rightarrow \infty, \Delta t \rightarrow 0} \sum_{j=1}^{n}\left(f\left(x_{t_{j}}\right)+f\left(x_{t_{j-1}}\right)\right) \cdot\left(x_{t_{j}}-x_{t_{j-1}}\right)
\end{aligned}
$$

where the symbol $\circ$ is commonly added as a notation to distinguish between Itô and Stratonovich interpretations. For our analysis it is important to observe that the Stratonovich integral is time-antisymmetric, and also that

$$
\int_{0}^{T} \nabla g\left(x_{t}\right) \circ \mathrm{d} x_{t}=g\left(x_{T}\right)-g\left(x_{0}\right)
$$

Relation between Itô and Stratonovich. For a scalar $f$, it is easily found from (A1) that

$$
\int_{0}^{T} f\left(x_{t}\right) \circ \mathrm{d} x_{t}=\int_{0}^{T} f\left(x_{t}\right) \mathrm{d} x_{t}+\int_{0}^{T}(D \nabla f)\left(x_{t}\right) \mathrm{d} t
$$

(and analogously for vectors). Using that $x_{t}$ solves the Itô-stochastic equation (8), that Stratonovich integral further explicitly equals to

$$
\int_{0}^{T} f\left(x_{t}\right) \circ \mathrm{d} x_{t}=\int_{0}^{T}[f \chi(F-\nabla U)+\nabla \cdot(f D)]\left(x_{t}\right) \mathrm{d} t+\int_{0}^{T} f\left(x_{t}\right) \sqrt{2 D\left(x_{t}\right)} \mathrm{d} B_{t}
$$

Observe that the last Itô-integral has mean zero since the integrand evaluated at each mesh point $x_{t_{j-1}}$ and the increment $B_{t_{j}}-B_{t_{j-1}}$ of the Brownian motion are mutually independent, and the latter has zero mean. Hence, the mean value of the Stratonovich integral (A6) is

$$
\begin{aligned}
\left\langle\int_{0}^{T} f\left(x_{t}\right) \circ \mathrm{d} x_{t}\right\rangle_{\mu_{0}} & =\left\langle\int_{0}^{T}[f \chi(F-\nabla U)+\nabla \cdot(f D)]\left(x_{t}\right) \mathrm{d} t\right\rangle_{\mu_{0}} \\
& =\int_{0}^{T} \mathrm{~d} t \int \mu_{t}[f \chi(F-\nabla U)+\nabla \cdot(f D)] \mathrm{d} x \\
& =\int_{0}^{T} \mathrm{~d} t \int f\left[\chi(F-\nabla U) \mu_{t}-D \nabla \mu_{t}\right] \mathrm{d} x
\end{aligned}
$$

The first equality is obtained by taking the mean of (A7). The second equality uses that $\mu_{t}$, defined by the FokkerPlanck equation (9), is the evolved density at time $t$ when starting from $\mu_{0}$ at time zero. Therefore $x_{t}$ is there distributed according to $\mu_{t}$. The final equality is from a partial integration. That proves the equality (11). 
The path of a particle subject to the stochastic equation (8) is completely determined by the initial condition and the realization of the Brownian motion $B_{t}$. Since the latter is a standard Gaussian process, we can integrate out the noise to obtain a path-integral representation. We restrict here to a simple heuristic argument which goes as follows.

To find the probability for the particle to pass through (the neighborhood of) a discrete set of points $\omega=\left(x_{0}, t_{0}=\right.$ $\left.0 ; x_{1}, t_{1} ; \ldots ; x_{n}, t_{n}=T\right)$, and having already in mind the limit $n \rightarrow \infty$, we use that each increment $\Delta x_{j}=x_{j}-x_{j-1}$ is by (8) in a one-to-one correspondence with an increment of the Brownian motion, which is $\left(2 D\left(x_{j-1}\right)\right)^{-1 / 2}\left[\Delta x_{j}-\right.$ $\left.(\chi F-\chi \nabla U+\nabla \cdot D) \Delta t_{j}\right]$. Since the latter increments are independent standard Gaussian variables, we immediately obtain the discrete path-distribution (or its density with respect to the $(n+1)$-product of flat distributions):

$$
\begin{aligned}
& \mathrm{d} \boldsymbol{P}_{\mu_{0}}(\omega) \simeq \mu\left(x_{0}\right) \delta x_{0} \prod_{j=1}^{n}\left[4 \pi D\left(x_{j-1}\right) \Delta t_{j}\right]^{-\frac{1}{2}} \\
& \times \exp \left\{-\frac{1}{4 \Delta t_{j}}\left[\Delta x_{j}-(\chi F-\chi \nabla U+\nabla \cdot D)\left(x_{j-1}\right) \Delta t_{j}\right]\right. \\
&\left.\quad \cdot D^{-1}\left(x_{j-1}\right)\left[\Delta x_{j}-(\chi F-\nabla U+\nabla \cdot D)\left(x_{j-1}\right) \Delta t_{j}\right]\right\} \delta x_{j}
\end{aligned}
$$

where the initial point $x_{0}$ is sampled from an initial distribution $\mu_{0}$. A useful representation employs the reference process

$$
\mathrm{d} x_{t}=\nabla \cdot D\left(x_{t}\right) \mathrm{d} t+\sqrt{2 D\left(x_{t}\right)} \mathrm{d} B_{t}
$$

with the initial point sampled from the flat distribution; note the latter (unnormalizable in general) distribution is stationary. Its path-distribution $\boldsymbol{P}^{0}$ is simply obtained from (B1) by putting $F=\nabla U=0$ and $\mu=1$. Comparing both path-distributions and passing to the limit $n \rightarrow \infty, \Delta t \rightarrow 0$, we obtain a Girsanov-formula for diffusions:

$$
\begin{aligned}
\frac{\mathrm{d} \boldsymbol{P}_{\mu}}{\mathrm{d} \boldsymbol{P}^{0}}(\omega)=\mu\left(x_{0}\right) \exp \left\{-\frac{\beta}{4} \int_{0}^{T}[(F-\nabla U) \cdot \chi(F-\nabla U)]\left(x_{t}\right) \mathrm{d} t\right. & \left.-\frac{1}{2} \int_{0}^{T}[(\nabla \cdot \chi) \cdot(F-\nabla U)]\left(x_{t}\right) \mathrm{d} t+\frac{\beta}{2} \int_{0}^{T}(F-\nabla U)\left(x_{t}\right) \cdot \mathrm{d} x_{t}\right\}
\end{aligned}
$$

where an Itô integral comes out by construction. Using the relation (A6) to replace that integral with a Stratonovich one, we finally arrive at formulas (15)-(17). A rigorous derivation can e.g. be found in [30].

[1] A. Einstein, Ann. d. Phys., 17, 549 (1905).

[2] L. Onsager and S. Machlup, Phys. Rev. 91, 1505 (1953).

[3] L. Bertini, A. De Sole, D. Gabrielli, G. Jona-Lasinio, and C. Landim, Phys. Rev. Lett. 87, 040601 (2001); J. Stat. Phys. 107, 635-675 (2002).

[4] R. Kubo, K. Matsuo, and K. Kitahara, J. Stat. Phys. 9, 51-95 (1973).

[5] D. J. Evans, E. G. D. Cohen, and G. P. Morriss, Phys. Rev. Lett. 71, 2401 (1993).

[6] G. Gallavotti and E. G. D. Cohen, Phys. Rev. Lett. 74, 2694 (1995); J. Stat. Phys. 80, 931 (1995).

[7] L. Bertini, A. De Sole, D. Gabrielli, G. Jona-Lasinio, and C. Landim, Phys. Rev. Lett. 94, 030601 (2005).

[8] T. Bodineau and B. Derrida, Phys. Rev. Lett. 92, 180601 (2004).

[9] B. Derrida, cond-mat/0703762v1.

[10] S. Pilgram, A. N. Jordan, E. V. Sukhorukov, and M. Büttiker, Phys. Rev. Lett. 90, 206801 (2003).

[11] C. Flindt, T. Novotný, and A. P. Jauho, Europhys. Lett. 69, 475-481 (2005).

[12] C. Maes and M. H. van Wieren, Phys. Rev. Lett. 96, 240601 (2006).

[13] W. De Roeck and C. Maes, cond-mat/0610369.

[14] C. Maes and K. Netočný, J. Math. Phys. 48, 053306 (2007).

[15] S. Bruers, C. Maes, and K. Netočný, cond-mat/0701035.

[16] C. Maes and K. Netočný, cond-mat/0705.2344.

[17] N.V. Krylov, Introduction to the Theory of Diffusion Processes (Translations of Mathematical Monographs), American Mathematical Society (1995).

[18] M. D. Donsker and S. R. Varadhan, Comm. Pure Appl. Math., 28:1-47 (1975).

[19] A. Dembo and O. Zeitouni, Large Deviations Techniques and Applications, Springer-Verlag, New York, Inc (1998). 
[20] S. R. S. Varadhan, Large Deviations and Entropy, In: Entropy, Eds. A. Greven, G. Keller and G. Warnecke, Princeton University Press, Princeton and Oxford (2003).

[21] W. De Roeck, C. Maes, and K. Netočný, J. Stat. Phys. 123:571-584 (2006).

[22] C. Maes and K. Netočný, J. Stat. Phys. 110, 269 (2003).

[23] C. Maes, in: Séminaire Poincaré 2, 29 (2003), Eds. J. Dalibard, B. Duplantier, and V. Rivasseau, Birkhäuser (Basel).

[24] R. Chetrite and K. Gawedzki, cond-mat/0707.2725.

[25] More precisely, that asymptotic law needs to be understood in the sense $\lim _{\epsilon \rightarrow 0} \lim _{T \rightarrow \infty}(1 / T) \log \boldsymbol{P}\left(\bar{\mu}_{T} \stackrel{\epsilon}{=} \mu\right)=I(\mu)$, where $\bar{\mu}_{T} \stackrel{\epsilon}{=} \mu$ stands for a neighborhood of $\mu$ in a weak metrizable topology (see e.g. [19], appendix D.2 for more details).

[26] J. R. Dorfman, An Introduction to Chaos in Nonequilibrium Statistical Mechanics, Cambridge University Press (1999).

[27] J. R. Dorfman and P. Gaspard, Physical Review E 51, 28 (1995).

[28] I. Prigogine, Introduction to Non-Equilibrium Thermodynamics, Wiley- Interscience, New York (1962).

[29] C. W. Gardiner, Handbook of Stochastic Methods for Physics, Chemistry and The Natural Sciences, Springer, Berlin (2004).

[30] R. S. Lipster and A. N. Shiryayev, Statistics of Random Processes I \& II, Springer-Verlag, New York (1977). 